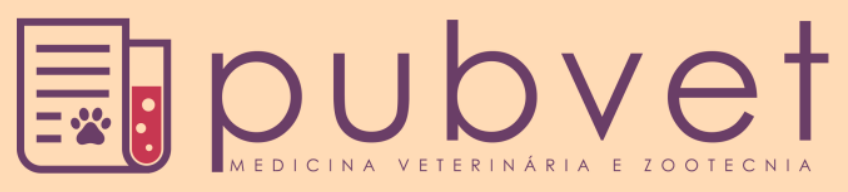

HTTP://DX.DOI.ORG/10.22256/PUBVET.V11N12.1263-1273

\title{
Geração de energia elétrica e viabilidade técnico-econômica de um biodigestor no setor hospitalar ${ }^{1}$
}

\author{
Kennedy Luiz Souza do Nascimento ${ }^{2}$, Stoécio Malta Ferreira Maia ${ }^{3}$, Sarah Jacqueline \\ Cavalcanti da Silva ${ }^{4}$, Elton Lima Santos ${ }^{4}$
}

${ }^{1}$ Parte da Dissertação de Mestrado do primeiro autor.

${ }^{2}$ Mestre em Energia da Biomassa da Universidade Federal de Alagoas, Centro de Ciências Agrárias. Rio Largo-AL, Brasil. E-mail: nascimentokennedy@hotmail.com

${ }_{3}^{3}$ Professor (a) da Instituto Federal de Alagoas, Campus Marechal Deodoro. Marechal Deodoro-AL, Brasil.

${ }^{4}$ Professor (a) da Universidade Federal de Alagoas, Centro de Ciências Agrárias. Rio Largo-AL, Brasil.

*Autor para correspondência, E-mail: elton.santos@ceca.ufal.br

\begin{abstract}
RESUMO. A construção de hospitais se transformou e evoluiu sensivelmente nas últimas décadas, sendo visto como um processo industrializado, com aumento em escala, o que gera grandes quantidades de resíduos, causando grandes problemas ambientais. Dentro deste contexto, faz-se necessária uma nova disposição para os resíduos produzidos (biomassa) e sua reutilização. Seguindo técnicas apropriadas, surge como opção o uso do biodigestor, o qual permite que os resíduos hospitalares sejam tratados podendo gerar energia elétrica e/ou gás natural através do biogás e, ainda, possibilitam o emprego do efluente residual como fertilizante de solo. O presente estudo teve como objetivo avaliar o potencial de geração de energia e a viabilidade econômica e ambiental da adoção de um biodigestor no Hospital Geral do Estado de Alagoas (HGE). Para tal realizou-se um levantamento da biomassa residual produzida no HGE, do consumo, dos custos e da oscilação da potência elétrica e utilizaram-se equações para a obtenção dos valores de produção energética, a viabilidade econômica e ambiental. Os resultados obtidos mostraram que o hospital gera 20.914,40 kg/ano-1 de resíduos alimentares, com o potencial de geração de 840 m3/ano-1 de biogás, uma produção de energia elétrica de 5.352 $\mathrm{kWh} / \mathrm{ano}-1$. Isso resulta em um custo do equipamento que foi de $\mathrm{R} \$ 80.598,00$. Do ponto de vista econômico, a adoção do biodigestor no HGE não se mostrou viável, visto que a energia elétrica gerada por este sistema apresentaria o custo de $\mathrm{R} \$ / \mathrm{KWh} 1,45$, enquanto que o valor pago a Eletrobrás é de $\mathrm{R} \$ / \mathrm{KWh} 0,24$.
\end{abstract}

Palavras chave: Biogás, eletricidade, hospital

\section{Electric energy generation and technical - economic viability of a biodigester in the hospital sector}

ABSTRACT. The construction of hospitals has transformed and evolved sensibly in the last decades, being seen as an industrialized process, with increase in scale, which generates large amounts of waste, causing great environmental problems. Within this context, a new provision is needed for the waste produced (biomass) and its reuse. Following the appropriate techniques, the use of the biodigester, which allows hospital waste to be treated, can generate electricity and/or natural gas through the biogas and allows the use of the residual effluent as a soil fertilizer. The present study aimed to evaluate the potential of energy generation and the economic and environmental viability of the adoption of a biodigester at the General Hospital of the State of Alagoas (HGE). For this, a study of the residual biomass produced in the HGE, consumption, and costs, electric power oscillation was carried out, and equations were used to obtain the valuesof energy production, 
economic and environmental viability. The results show that the hospital generates $20,914.40 \mathrm{Kg} /$ year $^{-1}$ of food waste, with a potential of $840 \mathrm{~m}^{3} /$ year $^{-1}$ of biogas, producing electricity of 5,352 kWh / year-1. This results in a cost of equipment that was $\mathrm{R} \$ 80,598.00$. From an economic point of view, the adoption of the biodigester in the HGE was not feasible, since the electric energy generated by this system would present the cost of R $\$$ / $\mathrm{KWh} 1.45$, while the amount paid to Eletrobrás is $\mathrm{R} \$$ / KWh 0.24 .

Key words: Biogas, electricity, hospital

\section{Generación de energía eléctrica y viabilidad técnico-económica de un biodigestor en el sector hospitalario}

RESUMEN. La construcción de hospitales se ha transformado y evolucionado sensiblemente en las últimas décadas, siendo visto como un proceso industrializado, con aumento en escala, lo que genera grandes cantidades de residuos, causando grandes problemas ambientales. En este contexto, se hace necesaria una nueva disposición para los residuos producidos (biomasa) y su reutilización. Siguiendo técnicas apropiadas, surge como opción el uso del biodigestor, el cual permite que los residuos hospitalarios sean tratados pudiendo generar energía eléctrica y / o gas natural a través del biogás y, además, posibilitan el empleo del efluente residual como fertilizante de suelo. El presente estudio tuvo como objetivo evaluar el potencial de generación de energía y la viabilidad económica y ambiental de la adopción de un biodigestor en el Hospital General del Estado de Alagoas (HGE). Para ello se realizó un levantamiento de la biomasa residual producida en el HGE, del consumo, de los costos y de la oscilación de la potencia eléctrica y se utilizaron ecuaciones para la obtención de los valores de producción energética, la viabilidad económica y ambiental. Los resultados obtenidos mostraron que el hospital genera $20.914,40 \mathrm{~kg} / \mathrm{año}^{-1}$ de residuos alimentarios, con el potencial de generación de $840 \mathrm{~m}^{3}$ / año-1 de biogás, una producción de energía eléctrica de $5.352 \mathrm{kWh} / \mathrm{año}^{-1}$. Esto resulta en un costo del equipo que fue de $\mathrm{R} \$ 80.598,00$. Desde el punto de vista económico, la adopción del biodigestor en el HGE no se mostró viable, ya que la energía eléctrica generada por este sistema presentaría el costo de R $\$$ / KWh 1,45, mientras que el valor pagado a Eletrobrás es de R \$ KWh 0,24.

Palabras clave: Biogás, electricidad, hospital

\section{Introdução}

A realidade dos hospitais em relação ao gerenciamento dos impactos ambientais tem sido um assunto amplamente questionado por entidades ambientais e pesquisadores, principalmente devido às consequências e dificuldades existentes quanto ao sistema de tratamento de resíduos gerados que deveria ser utilizado (Santos et al., 2017) e também pela falta de conhecimento de profissionais da área (Pfitscher et al., 2006).

É notório que o aumento da quantidade de pacientes nas clinicas de saúde e hospitais, associadas às deficiências de estruturas organizacionais fizeram surgir à necessidade da criação de processos e organizações capazes de reciclar e tratar os resíduos hospitalares e outros resíduos orgânicos provenientes destes lugares (Pfitscher et al., 2006).
A gestão dos resíduos de um Hospital é complexa e seu êxito depende em grande medida, da mudança de hábitos das pessoas que utilizam e trabalham no hospital. Na maioria dos hospitais de grande porte, os padrões operacionais requerem um consumo de energia significativa para aquecimento, controles de temperatura, umidade, iluminação, ventilação e outros processos clínicos. Isto implica em grandes custos financeiros e emissões de gases de efeito estufa (RGHVS, 2015). Os estabelecimentos de saúde também podem reduzir progressiva e significativamente as suas emissões de gases de efeito estufa e seus custos energéticos utilizando formas alternativas de energia renovável, tais como o da biomassa orgânica produzida no próprio hospital, pelo uso de biodigestor. Entende-se também que a avaliação de aspectos técnicos e econômicos da implantação e operação de biodigestores na produção de biogás, pode contribuir para a formulação de políticas públicas voltadas ao 
desenvolvimento local de forma sustentável. Ainda, apoiar a tomada de decisão quanto à implantação dessas tecnologias para o segmento da geração de energia em pequenas escalas nos hospitais.

O presente trabalho tem como objetivo avaliar o potencial de geração de energia e a viabilidade técnica e econômica da adoção de um biodigestor no Hospital Geral do Estado de Alagoas.

\section{Material e Métodos}

O estudo foi realizado no Hospital Geral do Estado Prof. Osvaldo Brandão Vilela (HGE), localizado na cidade de Maceió, no Estado de Alagoas, Brasil. Atualmente, o hospital conta com 278 leitos.

O Hospital Geral é dividido por áreas: Área Vermelha, destinada à pacientes graves; Área Amarela, pacientes em observação, Área Azul, destinada a pacientes menos grave e a Área Verde, destinada aos pacientes internados. Ao todo são 15 mil $\mathrm{m}^{2}$ de área física construída.

O estudo contemplou três etapas: a) levantamento da demanda de energia geral e quantidades consumidas em KWh no período de junho de 2013 a maio de 2014; b) quantificação dos resíduos alimentares gerados no Hospital Geral do estado de Alagoas (HGE), no período de março a setembro de 2014 e cálculo do potencial de geração de biogás; e c) no dimensionamento e a análise da viabilidade econômica da adoção de um biodigestor.

O levantamento do consumo total de energia elétrica mensal e o detalhamento de carga elétrica nos setores UTI, centro cirúrgico e cardiologia foram baseados no consumo mensal de energia elétrica em KWh do HGE, obtido no período de um ano (junho de 2013 a maio de 2014), a partir das contas de energias fornecidas pelo setor de contas da Secretaria de Saúde do Estado de Alagoas.

Os consumos detalhados dos setores de UTI, Centro Cirúrgico e Cardiologia foram determinados por meio do uso do equipamento Minipa $^{\circledR}$ (da empresa Brasnergy ${ }^{\circledR)}$ ) que possibilita analisar as variações dos sistemas elétricos especifico ou geral. O aparelho visa uma leitura dos parâmetros elétricos e as suas anomalias relacionadas às potências e tensões das cargas interligadas no quadro elétrico, é um equipamento para mensuração de operações em cargas elétricas, com analises de distúrbio de energia que podem envolver tensão, corrente ou frequência. As leituras foram realizadas em horas alternadas de 03 e 05 de abril de 2014, com parâmetros de dados conforme utilização de cargas (Equipamentos). O levantamento da quantidade de resíduos alimentares provenientes do hospital se deu durante o período de 01 de agosto a 31 de outubro de 2014. Foram quantificados os resíduos gerados nos almoços e jantares de funcionários e acompanhantes da unidade hospitalar e do serviço de nutrição, durante o período de levantamentos dos resíduos foi utilizado uma balança com capacidade de $100 \mathrm{~kg}$.

Os resíduos eram pesados diariamente em dois turnos (tarde e noite) proporcionando os resultados em nível de quantitativos, gerados por dia no HGE, em restos de alimentos.

$\mathrm{O}$ potencial de geração de energia elétrica foi estimado considerando a relação de $1,0 \mathrm{~m}^{3} \mathrm{de}^{\mathrm{CH}_{4}}$ gera em média $1,5 \mathrm{~m}^{3}$ de gás de cozinha ou 6,4 KWh de eletricidade (Deganutti et al., 2002, Medeiros et al., 2015).

Para o cálculo da estimativa de produção do biogás, utilizou-se como base de que os resíduos alimentares que eram, na sua maior partem de restos vegetais, ao qual se utilizou a equação abaixo, segundo Beduschi et al. (2009):

\section{$\mathrm{PB}=\mathrm{RV} \times \mathrm{CR}$}

Onde:

$P B=$ Produção de biogás $\left(\mathrm{m}^{3}\right) R V=$ Resíduos vegetais $(\mathrm{kg})$ $C R=$ Coeficiente dos resíduos vegetais em $\mathrm{m}^{3}$. $\mathrm{e}$

\section{$\mathrm{VB}=\mathrm{VC} \mathrm{x}$ TRH}

Onde,

$V B=$ Volume do biodigestor

$V C=$ Volume de Carga diária

$T R H=$ Tempo de retenção hidráulica

Para o dimensionamento do biodigestor e análise de viabilidade econômica no presente trabalho, foi proposto o biodigestor do tipo modelo indiano, por se tratar do tipo mais recomendado para se trabalhar com materiais que exalam fortes odores, pois o selo d'água isola a mistura em fermentação do meio externo.

$\mathrm{O}$ biodigestor indiano se caracteriza por apresentar grande capacidade de armazenamento de biogás, facilitando seu uso em picos de consumo. Além disso, esse modelo, construído em formato de poço, onde ocorre a digestão da biomassa, é coberto por tampa cônica regulável, 
permitindo o controle da emissão do gás metano (Batista, 1981).

O biodigestor proposto é feito em formação metálica e composto de um reservatório de $3 \mathrm{~m}$ de diâmetro da parte superior e $3 \mathrm{~m}$ parte inferior, com $3 \mathrm{~m}$ de altura. Uma barreira de divisória a uma altura de $70 \mathrm{~cm}$, além de um cilindro de movimento de $2,80 \mathrm{~m}$ e um eixo central de $6,30 \mathrm{~m}$ fixo na parte superior e central na divisória de barreira chamada de tubo de guia, para a campânula metálica de deposito de gás foram usados.

A análise econômica do empreendimento consiste em fazer estimativas de todo o gasto financeiro envolvido com o investimento inicial, operação e manutenção e receitas geradas durante um determinado período de tempo. O Payback, ou seja, a avaliação do retorno do investimento descreve quanto tempo será necessário para que o capital investido inicialmente seja recuperado. O Payback foi calculado conforme a expressão:

$$
\begin{aligned}
& \text { Inv. Inicial } \\
& \mathrm{PB}=\sum \mathrm{FC} \text { ano }
\end{aligned}
$$$$
\text { Onde: }
$$

Inv. Inicial = custo do investimento inicial, em $R \$$. $F C=$ fluxo de caixa ao ano, em $R \$$.

Algumas premissas foram colocadas, como: os resíduos provenientes do restaurante do hospital são recolhidos por uma empresa prestadora de serviço, tais resíduos são colocados após as refeições dentro de contêineres até o término de cada período de alimentação, tanto para acompanhantes e funcionários.

O compactador da empresa terceirizada do HGE permanece no local a ser recolhido três vezes por semana. Os resíduos são compactados no contêiner e depois fechado para o transporte até o Centro de Tratamento de Resíduos (CTR), a cerca de $85 \mathrm{~km}$, e a cada 3 dias é abastecido com 280 litros de óleo diesel. Já os dados de consumo de energia elétrica, foram obtidos das contas mensais pagas pelo HGE. Todos os dados obtidos foram organizados em gráficos e tabelas, e avaliados de forma descritiva.

\section{Resultados e Discussão}

O levantamento do consumo e dos valores financeiros pagos, registrado pela fornecedora pública de energia elétrica, ELETROBRÁS, foi adquirido diretamente de leituras manuais no quadro de distribuição do Hospital Geral do
Estado no período de 25 de junho de 2013 a 27 de maio de 2014 e estão expressos na Tabela 1.

Tabela 1. Consumo de eletricidade e valores pagos no período de junho de 2013 a maio de 2014 no Hospital Geral do Estado de Alagoas.

\begin{tabular}{lcc}
\hline Meses/ano & Consumo (kWh) & Valores pagos (R\$) \\
\hline Jun./2013 & 244.533 & $52.784,81$ \\
Jul./2013 & 235.956 & $51.468,68$ \\
Ago./2013 & 219.517 & $49.999,35$ \\
Set./2013 & 237.138 & $51.901,61$ \\
Out./2013 & 249.112 & $64.250,81$ \\
Nov./2013 & 257.139 & $64.307,74$ \\
Dez./2013 & 257.698 & $66.678,02$ \\
Jan./2014 & 316.550 & $78.734,27$ \\
Fev./2014 & 370.066 & $89.288,17$ \\
Mar./2014 & 366.502 & $89.573,33$ \\
Abr./2014 & 326.230 & $81.383,96$ \\
Mai./2014 & 374.419 & $89.883,66$ \\
\hline Soma & 3.454 .860 & $835.254,41$ \\
Média & 287.905 & $69.604,53$ \\
DP & $\pm 229,79$ & $\pm 14.383,019$ \\
CV,\% & 19,46 & 23,50 \\
\hline
\end{tabular}

Observa-se, portanto, que a maior potência geral consumida pelo hospital foi no mês de maio de 2014 com $374.419 \mathrm{kWh}$ e com uma despesa de $\mathrm{R} \$ 89.883,66$. Em contrapartida, a menor potência geral consumida pelo HGE foi verificada no mês de agosto de 2013, com $219.517 \mathrm{kWh}$, gerando assim um custo mensal de $\mathrm{R} \$ 49.999,35$. No período de um ano o HGE consumiu o total de 3.454.860 kWh, resultando em um consumo médio mensal de $287.905 \mathrm{kWh}$ e um coeficiente de variação igual a $19,4 \%$. No que se refere ao custo deste consumo de energia, o HGE pagou a ELETROBRÀS nestes 12 meses o montante de $\mathrm{R} \$$ 835.254,41, com um custo médio mensal de $\mathrm{R} \$$ 69.604,53.

Embora a questão da eficiência energética e a questão da sustentabilidade impactem o consumo, certamente as políticas de inclusão social e de redução da desigualdade levarão à necessidade de se ampliar muito a disponibilidade de energia no País. Pode-se antever que o aumento da população mundial, principalmente nos países em desenvolvimento, levará ao crescimento da demanda por habitação (programa minha casa minha vida, por exemplo). Da mesma forma, ao aumento da demanda do setor industrial e de transporte que serão os principais vetores para o crescimento do consumo mundial de energia (Bueno, 2013). 
É natural o alto consumo de energia elétrica observado, visto que o HGE é a maior unidade de saúde do Estado de Alagoas, onde são atendidos pacientes de todo o Estado e no qual o seu funcionamento deve ser realizado durante as 24 horas do dia em todos os dias da semana. Entretanto, a avaliação criteriosa dos custos advindo desse alto consumo e a análise de alternativas para minimizar esses custos deve ser buscada incessantemente pelos gestores e técnicos responsáveis, pois, os custos com energia elétrica são consideráveis. Deste modo a economia nos custos poderá ser um suporte importante para investimento em outras áreas carentes do hospital.

É frequente e de conhecimento geral as notícias sobre a instabilidade de preços e qualidade da energia elétrica da rede de distribuição pública no estado de Alagoas e no Brasil. Isso ocorre devido a vários fatores como intempéries do tempo, maior uso das termoelétricas, problemas de transmissão, acidentes etc. Em hospital de alta complexidade, como é o caso do HGE, o fornecimento de energia elétrica não pode ser limitado, visto que muitos pacientes dependem de aparelhos ligados permanentemente para a sua sobrevivência. Desta forma, mesmo que haja oscilações no preço da energia elétrica fornecida, o Hospital não deixará de consumir de acordo com a sua necessidade. Desta forma, deve pensar que outras formas de fornecimento de energia elétrica, mais baratas, podem ser bem interessantes.

Grande parte das diferenças em relação ao consumo de energia elétrica encontrado no presente estudo, entre os diferentes meses se dá pelas diferenças no uso dos aparelhos, no qual alguns demandam alta potência para uso. Desta forma, os grandes consumidores de energia de uma unidade hospitalar são os aparelhos utilizados para fazer exames e tratamentos radioativos. Um estudo realizado em uma auditoria energética no Hospital "San Juan de Dios", na cidade de Estelí, na Nicaraguá verificaram que as falhas e problemas de potência dos equipamentos poderiam superar em até $75 \%$ os custos com a energia elétrica. Esses autores ainda relatam o alto custo com energia elétrica encontrada é um indicador direto de que é necessário um plano de medidas para melhorar essa situação.

No levantamento realizado no HGE, os custos anuais com a energia elétrica são de $R \$$ 835.254,41; considerado um valor alto e bastante expressivo, visto que é considerado como um gasto necessário, revelando assim, de forma cada vez mais imprescindível a busca por uma alternativa para diminuir esses valores.

A escolha dos setores estudados se deu por serem considerado de extrema importância no tratamento de pessoas em situação gravíssima e que não é permitida a interrupção, seja total ou parcial, do potencial energético. Para isto foi utilizado à distribuição de energia que compõem os três setores interligados nas fases F1, F2 e F3 dos barramentos do quadro elétrico.

Tabela 2. Levantamento do consumo das tensões e potências do centro cirúrgico, UTI e setor de cardiologia do Hospital Geral do Estado de Alagoas.

\begin{tabular}{|c|c|c|c|}
\hline \multicolumn{2}{|c|}{ Data/hora } & Tensão máxima & Potência máxima \\
\hline \multicolumn{4}{|c|}{ 03/04/2014 } \\
\hline & $20 \mathrm{~h} 36 \mathrm{~min}$ & 169.200 & 120.000 \\
\hline & $22 \mathrm{~h} 06 \mathrm{~min}$ & 148.300 & 99.050 \\
\hline & $22 \mathrm{~h} 36 \mathrm{~min}$ & 142.600 & 72.040 \\
\hline & $23 \mathrm{~h} 06 \mathrm{~min}$ & 178.600 & 129.000 \\
\hline & $23 \mathrm{~h} 51 \mathrm{~min}$ & 125.000 & 82.570 \\
\hline Média & & & 100.532 \\
\hline $\mathrm{CV}, \%$ & & & 21,45 \\
\hline \multicolumn{4}{|c|}{$04 / 04 / 2014$} \\
\hline & 06h06min & 184.600 & 134.600 \\
\hline & $02 \mathrm{~h} 06 \mathrm{~min}$ & 145.700 & 93.690 \\
\hline & 04h06min & 113.600 & 83.180 \\
\hline & $10 \mathrm{~h} 06 \mathrm{~min}$ & 187.500 & 139.600 \\
\hline & $16 \mathrm{~h} 06 \mathrm{~min}$ & 197.400 & 157.000 \\
\hline Média & & & 121.614 \\
\hline $\mathrm{CV}, \%$ & & & 23,26 \\
\hline \multicolumn{4}{|c|}{ 05/04/2012 } \\
\hline & $00 \mathrm{~h} 06 \mathrm{~min}$ & 81.980 & 48.230 \\
\hline & $02 \mathrm{~h} 06 \mathrm{~min}$ & 125.100 & 67.880 \\
\hline & 03h06min & 128.700 & 73.160 \\
\hline & $05 \mathrm{~h} 21 \mathrm{~min}$ & 131.500 & 77.820 \\
\hline & 07h06min & 54.000 & 32.360 \\
\hline Média & & & 59.890 \\
\hline $\mathrm{CV}, \%$ & & & 28,48 \\
\hline
\end{tabular}

$\mathrm{CV}$ - Coeficiente de variação.

De acordo, como os levantamentos realizados nos setores específicos do hospital foram verificados que a maior potência registrada no quadro elétrico de comandos foi de $157.000 \mathrm{~W}$. A menor potência registrada no quadro elétrico de comandos dos setores específicos foi de 17.180W. A variação da potência na UTI, cardiologia e centro cirúrgico com dados registrado em 139 leituras (Tabela 2).

Diante da análise realizada na potência, verificou-se a necessidade de estabilidade no 
fornecimento do potencial energético. Portanto observou-se que no HGE grandes oscilações entre a tensão e a potência máxima no fornecimento de energia elétrica.

Assim, quanto maior a necessidade da estabilidade no fornecimento de energia elétrica no hospital, maior terão que ser otimizados os protocolos de segurança, de forma a serem minimizados os riscos de interrupção da energia. Desta forma, estabelece-se como premente a busca de alternativas a utilização de energia elétrica proveniente da rede elétrica pública de abastecimento, ao qual, pode-se sugerir o uso de fontes renováveis, como o biogás oriundo de um biodigestor.

O levantamento da biomassa orgânica produzida no hospital contempla principalmente as refeições servidas aos funcionários do HGE e acompanhantes de pacientes. Os resultados são apresentados na Figura 1; e completam a quantidade média das refeições servidas, e a quantidade dos resíduos orgânicos gerados diariamente, nos meses de agosto a outubro.

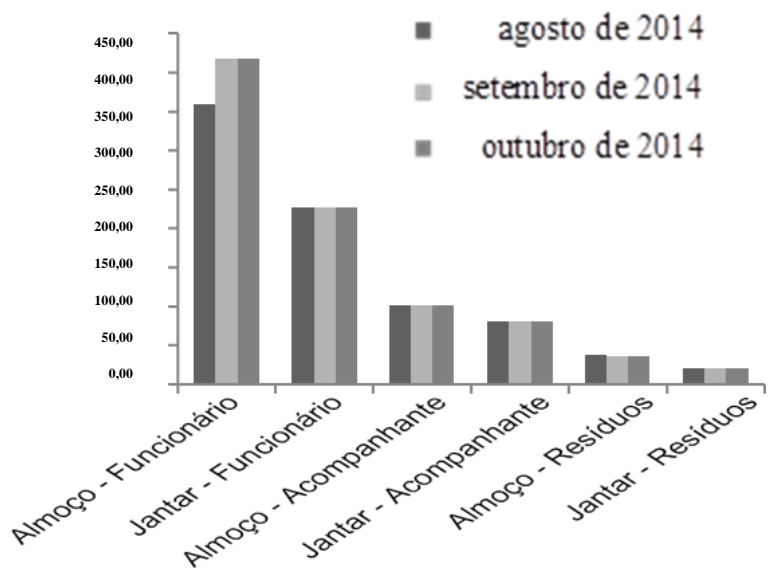

Figura 1. Biomassa orgânica produzida por as refeições servidas aos funcionários e acompanhantes de pacientes no Hospital Geral do Estado de Alagoas.

No mês de agosto foram ofertados a totalidade 11.070 almoços e 7.019 jantares para funcionários, da mesma forma que foram ofertados 3.121 almoços e 2.410 jantares para os acompanhantes dos pacientes totalizando 23.620 unidades alimentícias, os quais geraram uma média diária de biomassa residual de alimentos de $58.7 \mathrm{~kg}$.

No mês de setembro foram servidos 12.519 almoços e 6.784 jantares para os funcionários, enquanto que para os acompanhantes foram ofertados 4.068 almoços e 2.398 jantares. Sendo o total de almoços e jantares de funcionários e acompanhante do hospital no mês de setembro de 25.769 unidades alimentares. A média de almoços ofertados por dia foi de aproximadamente 417 e 101 refeições para funcionários e acompanhantes, respectivamente. A quantidade média de refeições noturnas ofertadas foi de 226 para os funcionários e 80 para os acompanhantes de pacientes, respectivamente. A média de resíduos alimentícios geradas por dia no HGE foi de $57 \mathrm{~kg}$.

Foram consumidos no mês de outubro 10.515 almoços e 6.880 jantares para funcionários, e 3.272 almoços e 3.026 jantares para os acompanhantes dos pacientes. Compondo um total de 23.693 refeições, entre almoços e jantares. A biomassa residual média por dia proveniente do almoço e jantar foi de $57 \mathrm{~kg}$ de restos alimentares no mês de outubro. Por dia também eram consumidos aproximadamente 181 refeições em média para os acompanhantes de pacientes e 590 refeições em média para os funcionários, incluindo o almoço e o jantar.

Verificaram-se os valores totais de biomassa de resíduos alimentares no HGE. Baseando-se nos três meses de amostragem, o que representa $25,0 \%$ do período de um ano, têm-se em média 1.742,8 $\mathrm{kg}$ mês $^{-1}$ (dados não mostrados) de resíduos provenientes do refeitório. Ressalta-se, portanto, que muito desses resíduos orgânicos poderiam ser mais bem utilizados, no que tange o seu aproveitamento. Assim, a falta de informações sobre o assunto faz com que, em muitos casos, os resíduos, ou seja, ignorados, ou recebam um tratamento com excesso de cuidado, onerando ainda mais os já combalidos recursos das instituições hospitalares, como é o caso do HGE.

Os resíduos alimentares são alvos frequentes de ações visando o seu uso nos processos de biodigestão anaeróbia como foi destacado por Sotti (2014). Este autor estudou a utilização das sobras das refeições de uma universidade na cidade de Ibiporã - PR e também do efluente proveniente da estação de tratamento de esgoto da cidade, alcançando sucesso no processo de digestão anaeróbia desses resíduos.

Entretanto, nos hospitais, o que pode parecer até contraditório, às destinações dos resíduos orgânicos são encaminhadas a aterros sanitários, podendo ser fontes futuras de infecções e locais multiplicadores de vetores de doenças. Geralmente, esses resíduos são compostos do descarte dos restos das refeições servidas a funcionários e acompanhantes e geram uma quantidade substancial de folhas, casca de frutas, 
legumes e restos de comida, poderiam ser coletados seletivamente e encaminhados para abastecer um ou mais biodigestores, tendo vários benefícios imediatos, como: geração de energia limpa, redução do consumo de GLP, diminuição dos resíduos e produção de biofertilizante de alta qualidade que poderia ser doado a agricultores da região ou ser utilizado como adubo orgânico na horta de escolas. Geralmente, os resíduos hospitalares e de instituições de saúde são as maiores fontes de problemas ambientais e de saúde pública. Assim, requer a disposição de um plano seguro de manejo desses resíduos (RGHVS, $\underline{2015}$ ).

Em relação ao destino dos resíduos provenientes do restaurante do Hospital Geral do Estado, pode-se constatar que todos os resíduos orgânicos são recolhidos por uma empresa terceirizada e armazenados em contêiner fechados, logo após o período do término da oferta das refeições. Destaque-se que não há separação dos resíduos provenientes da alimentação de acompanhantes dos pacientes e dos funcionários no hospital, permanecendo esses resíduos misturados até que sejam recolhidos posteriormente e levados para uma máquina compactador, pertencente a empresa terceirizada, onde permanecem até a chegada do caminhão específico para essa finalidade, sendo encaminhados para o Centro de Tratamento de Resíduos (CTR) de Maceió, localizado no bairro de Benedito Bentes, para tal o HGE arca com as despesas do serviço junto a uma empresa terceirizada.

Os estabelecimentos de saúde podem minimizar a geração de resíduos e as emissões de gases de efeito estufa por meio da utilização de biodigestores, compostagem, comprando produtos reutilizáveis ao invés de descartáveis e produtos reciclados, como também minimizando o transporte de resíduos. Uma vez que os resíduos oriundos dos estabelecimentos de saúde, se devidamente gerenciados, não deveriam causar nenhum impacto à saúde humana nem ao meio ambiente, fato que devido ao mau gerenciamento desses resíduos problemas podem ser causados (Nogueira, 1992).

A partir dos dados obtidos estima-se que em um dia o HGE produz aproximadamente $58,09 \mathrm{~kg}$ de resíduos alimentares, os quais podem ser racionalmente aproveitados para uso em um biodigestor. No entanto, o uso dos resíduos orgânicos do HGE para a produção de energia, deve-se ser implantada em conjunto com outras medidas de educação ambiental e manejo racional dos resíduos, para que auxiliem a otimização do sistema e o sucesso seja alcançado. Assim, devese desenvolver mecanismos, que enfatizam campanhas educativas e sócio ambientais de segregação dos resíduos e seu destino racional, tendo isto correlação direta com a diminuição dos custos operacionais de qualquer programa de eficiência energética e manejo dos resíduos orgânicos em um hospital. De acordo com Rodrigues et al. (2017) a maior parte dos resíduos orgânicos é composta por restos de vegetais. Considerando essa informação, formulou-se a $\underline{\text { Tabela } 3}$, em que se adotou que cada para kg deste tipo de resíduo gera em média $0,04 \mathrm{~m}^{3}$ de biogás (Metz, 2013), ou seja, para cada $1 \mathrm{~kg}$ de resíduo vegetal $=0,04 \mathrm{~m}^{3}$ de biogás e $1 \mathrm{~m}^{3}$ de biogás equivale a $6,4 \mathrm{kWh}$ ou $1,5 \mathrm{~m}^{3} \mathrm{GLP}$.

Tabela 3. Equivalência energética de $1 \mathrm{~m}^{3}$ de biogás em relação a outras fontes de energia.

\begin{tabular}{|c|c|c|c|c|}
\hline Energético & $\frac{\text { Catapan et }}{\underline{\text { al. }(2010)}}$ & $\frac{\text { Sganzerla }}{\underline{(1983)}}$ & $\frac{\text { Nogueira }}{\underline{(1992)}}$ & $\frac{\underline{\text { Santos }}}{\underline{(2000)}}$ \\
\hline Gasolina, L & 0,61 & 0,61 & 0,61 & 0,6 \\
\hline Querosene, L & 0,58 & 0,58 & 0,62 & - \\
\hline Diesel, L & 0,55 & 0,55 & 0,55 & 0,6 \\
\hline GLP, L & 0,45 & 0,45 & 1,43 & - \\
\hline Álcool, L & - & 0,79 & 0,8 & - \\
\hline Carvão mineral, $\mathrm{kg}$ & - & 0,74 & 0,74 & - \\
\hline Lenha, kg & - & 1,54 & 3,5 & 1.6 \\
\hline Eletricidade, $\mathrm{kWh}$ & 1.43 & 1.43 & - & 6,5 \\
\hline
\end{tabular}

Baseando-se na estimativa para a produção de biogás nos três meses avaliados e a avaliação de resíduos obtidos no HGE, foram estimados que nos meses de agosto, setembro e outubro poderiam ser produzidos 72,8, 68,3 e 68,1 $\mathrm{m}^{3}$ de biogás, respectivamente. Diariamente, estima-se produção total de biogás de aproximadamente $2,33 \mathrm{~m}^{3}$. Em relação à estimativa da produção de energia elétrica, baseou-se que: $1 \mathrm{~m}^{3}$ de biogás equivale a $6,4 \mathrm{kWh}$, sendo assim, nos meses de agosto, setembro e outubro, poderiam ser produzidos 465,$8 ; \quad 437,1$ e $435,6 \quad \mathrm{kWh}$, respectivamente. A energia elétrica produzida em 3 meses seria de $1.338 \mathrm{kWh}$, sendo cada mês 446 $\mathrm{kWh}$ em média e a produção de energia aproximada em um ano de $5.352 \mathrm{kWh}$. Ou seja, isso representa que a adoção do biodigestor poderia produzir $0,155 \%$ da energia consumia no HGE que é de $3.454 .860 \mathrm{kWh}$

Avaliando-se a estimativa da produção de gás 
de cozinha nos meses de agosto, setembro e outubro, obtém-se: $209 \mathrm{~m}^{3}$, e ao se considerar os dados de Nogueira (1992), em que cada metro cúbico de biogás equivale a $0,45 \mathrm{~kg}$ de gás, portanto nos três meses de produção de biogás e de 7,2 botijões de $13 \mathrm{~kg}$.

Para o dimensionamento do biodigestor foi levado em consideração à produção diária de resíduos teve uma variação de $35,15 \mathrm{~kg}$ a $72,01 \mathrm{~kg}$; portanto obtemos como referência um valor médio de $58,1 \mathrm{~kg}$ para o cálculo do volume diário do biodigestor, ou seja, 5.228,6 kg em 90 dias, o que equivale a $58,1 \mathrm{~kg}$ por média diária.

$\mathrm{O}$ projeto do equipamento que realizará o processo de digestão anaeróbica de nutrientes terá que ter condições controladas de: temperatura, água, alcalinidade, $\mathrm{pH}$ e ausência de oxigênio, resultando no gás metano e biofertilizante.

Segundo Lucas Júnior (2016), o tempo de retenção depende da finalidade para a qual o biodigestor foi construído. No presente estudo, foram utilizados resíduos alimentares com $20 \%$ de sólidos totais. Para atingir a mistura de $8 \%$ de sólidos, foram utilizados $58,09 \mathrm{~kg} / \mathrm{dia}$ (média) de resíduos alimentares e 90 litros de água (30\%), totalizando uma mistura de 148,09 L, com Tempo de Retenção Hidráulica (TRH) de 25 dias, capacidade de produção de $0,04 \mathrm{~m}^{3}$ de biogás por $\mathrm{kg}$ de resíduos, atingindo-se produção de $2,33 \mathrm{~m}$ de biogás por dia.

Os componentes para construção do biodigestor são apresentados na Tabela 4. Levando em consideração o modelo indiano com plataforma de produção para $10 \mathrm{~m}^{3} /$ dia de biogás. O sistema é dividido em 2 câmaras sendo uma para caixa de carga e a outra para caixa de descarga.

O protótipo do biodigestor apresenta-se todo em estrutura metálica, com fácil acesso ao sistema de carga, descarga e saída do gás. Da mesma forma, possibilita de maneira rápida sua manutenção e contem grades plataformas de segurança. Além disso, um compressor com sistema de capitação do biofertilizante para uma área de $20 \mathrm{~m}^{2}$ e com capacidade de produção de $10 \mathrm{~m}^{3}$ de biogás a partir do $25^{\circ}$ dia de abastecimento em resíduos orgânicos (restos de alimentos).

$\mathrm{O}$ biodigestor indicado foi o indiano. Este modelo tem sua cúpula geralmente feita de ferro ou fibra. Nesse tipo de biodigestor o processo de fermentação acontece mais rápido, pois aproveita a temperatura do solo que é pouco variável, favorecendo a ação das bactérias. Ocupa ainda pouco espaço e a construção por ser subterrânea ou externa, no caso de subterrânea dispensa o uso de reforços, tais como cintas de concreto.

Tabela 4. Levantamento dos materiais para fabricação do biodigestor no Hospital Geral do Estado de Alagoas.

\begin{tabular}{|c|c|c|c|c|}
\hline Item & Descrição & Unid. I & Unit. & Total, $\mathrm{R} \$$ \\
\hline 01 & Chapa Galv. 20 2x1, 20 & 06 & 63,00 & 378,00 \\
\hline 02 & Chapas alum. 16 2x1 & 04 & 162,00 & 648,00 \\
\hline 03 & Chapas xadrez 18 2x1 & 06 & 160,00 & 960,00 \\
\hline 04 & Tubos inox 1', & 04 & 96,00 & 384,00 \\
\hline 05 & Tubos inox 1,1/4', & 04 & 129,00 & 516,00 \\
\hline 06 & Cantoneira L $1 \times 1, / 8$ & 05 & 26,40 & 132,00 \\
\hline 07 & Cantoneira L 1x3/16 & 06 & 39,00 & 234,00 \\
\hline 08 & Cantoneira L $2.1 / 2 \times 1 / 4$ & 04 & 154,00 & 616,00 \\
\hline 09 & Telas alambrado $2 \mathrm{~m}$ & 10 & 30,00 & 300,00 \\
\hline 10 & Tubos Galv. 3" & 02 & 120,00 & 240,00 \\
\hline 11 & Tubos Galv. 2", & 02 & 85,00 & 170,00 \\
\hline 12 & Monômetro pressão & 01 & 180,00 & 180,00 \\
\hline 13 & Válvula & 01 & 210,00 & 210,00 \\
\hline 14 & Registros passagem & 02 & 95,00 & 190,00 \\
\hline 15 & Cimento $50 \mathrm{~kg}$ & 10 & 27,00 & 270,00 \\
\hline 16 & Areia (m) & 03 & 100,00 & 300,00 \\
\hline 17 & Brita $1(\mathrm{~m})$ & 04 & 120,00 & 480,00 \\
\hline 18 & Tijolos 06 furos & 500 & 0,90 & 450,00 \\
\hline 19 & Eletrodos Inox & 25 & 12,00 & 300,00 \\
\hline 20 & Eletrodos Galv. & 30 & 8,00 & 240,00 \\
\hline \multicolumn{4}{|c|}{ Total materiais } & $7.198,00$ \\
\hline \multicolumn{4}{|c|}{ Mão de Obra } & $8.500,00$ \\
\hline \multicolumn{4}{|c|}{$\begin{array}{l}\text { Compressor trifásico } 7,4 \text { hp, } 30 \text { pés, } 175 \text { libras, } \\
250 \text { litros. }\end{array}$} & $4.900,00$ \\
\hline
\end{tabular}

Gerador a Gás Mod.: Generac 125 KVA motor V6 Vortec $®$

$60.000,00$

Total geral

$80.598,00$

Entre diferentes testes econômicos existentes, o payback representa o tempo necessário para que a empresa recupere seu investimento inicial do projeto. É considerada por alguns economistas como uma técnica pouco sofisticada porque não leva em conta explicitamente o valor do dinheiro no tempo (correção financeira). No entanto, ainda é um dos índices mais utilizados para a avaliação da viabilidade econômica de investimentos do tipo da implantação de um biodigestor (Silva, 2012). Desta forma, o modelo sugerido por esse estudo possui custo para construção de $\mathrm{R} \$ \mathbf{8 0 . 5 9 8 , 0 0}$ (Tabela 4), com capacidade para produção de 10 $\mathrm{m}^{3}$ de biogás e ocupando uma área de $20 \mathrm{~m}^{2}$. O custo total do biodigestor contempla o conjunto motor/gerador composto por um motor de v6 e $125 \mathrm{KVA}$ de potência, $60 \mathrm{~Hz}$ em regime continuo 
com custo de implantação em torno de $\mathrm{R} \$$ $60.000,00$ e um compressor trifásico $7,4 \mathrm{hp}, 30$ pés 175 libras e 250 litros no valor de $\mathrm{R} \$ 4.900,00$.

Levou-se também em consideração a depreciação do conjunto biodigestor. A depreciação representa a desvalorização dos bens da propriedade, que perdem valor com o passar do tempo, os quais são denominados de bens depreciáveis. Os bens depreciáveis avaliados foram: equipamentos, máquinas e biodigestor. A Tabela 5 apresenta os dados obtidos.

Como forma de equiparar os dados dos componentes do biodigestor, adotou- se uma vida útil de 10 anos, assim, o valor específico para o biodigestor foi duplicado, desta forma, ao final de 10 anos será possível obter em torno de $\mathrm{R} \$$ $23.547,00$ com venda do material do biodigestor.

Tabela 5. Depreciação dos bens, avaliando-se o tempo de vida útil de 10 anos.

\begin{tabular}{lcccc}
\hline $\begin{array}{l}\text { Equipamento/ } \\
\text { Material }\end{array}$ & $\begin{array}{c}\text { Valor } \\
\text { inicial, R\$ útil, ano }\end{array}$ & $\begin{array}{c}\text { Vida } \\
\text { R } / \text { ano }\end{array}$ & $\begin{array}{c}\text { Depreciac̃alor } \\
\text { final } \$\end{array}$ \\
\hline Biodigestor & $15.698,00$ & 05 & 784,90 & $11.774,00$ \\
Grupo gerador & $60.000,00$ & 10 & $6.000,00$ & 0,00 \\
Compressor & $4.900,00$ & 10 & 490,00 & 0,00 \\
\hline Total & $80.598,00$ & & $7.274,00$ & $11.774,00$ \\
\hline Fonte:
\end{tabular}

Fonte: adaptado de Cervi et al. (2010).

Assim, a construção do conjunto biodigestor terá um custo líquido de $\mathrm{R} \$ 57.051,00$ levando em consideração os 10 anos. Somaram-se ao valor da construção os custos com a manutenção do conjunto, o qual foi de $\mathrm{R} \$ 2.060,00$ por ano (Tabela 6), tendo um custo de $\mathrm{R} \$ 20.600,00 \mathrm{em} 10$ anos.

Tabela 6. Manutenção do conjunto motor-gerador.

\begin{tabular}{lr}
\hline Componentes & $\begin{array}{r}\text { Custo anual de } \\
\text { Operação e } \\
\text { Manutenção (R\$) }\end{array}$ \\
\hline Troca do filtro de ar a cada 2.000 horas & 280,00 \\
Sistema de Refrigeração & 840,00 \\
Alternador Troca da correia a c/1.000 horas & 580,00 \\
Rolamento do gerador & 360,00 \\
\hline Total & $2.060,00$ \\
\hline
\end{tabular}

Portanto, o custo total do conjunto biodigestor seria a soma do custo liquido para construção (R\$ $57.051,00)$ com a manutenção ( $\mathrm{R} \$ 20.600,00)$, resultando em $\mathrm{R} \$ 77.651,00$. Assim, considerando o potencial de geração de energia elétrica do biodigestor que foi estimado em $5.352 \mathrm{Kwh} /$ ano, o custo médio do KWh seria de $\mathrm{R} \$ 1,45$.
O HGE consome anualmente $3.454 .860 \mathrm{kWh}$ de energia que corresponde ao valor de $\mathrm{R} \$$ $835.254,41$ por ano, o que resulta em uma tarifa de $0,24 \mathrm{R} \$ / \mathrm{kwh}$. Ou seja, o custo da energia gerada pelo biodigestor nestas condições é seis vezes maior que o custo da tarifa paga a Eletrobrás. É importante destacar que o biodigestor foi projetado para gerar até $10,0 \mathrm{~m} 3$ de biogás, sendo capaz, portanto, de produzir $23.000 \mathrm{KWh} / \mathrm{ano}$, o que reduziria o custo do KWh para $\mathrm{R} \$ 0,34$, se aproximando da tarifa paga atualmente a Eletrobrás. Obviamente, seria necessário, no entanto, buscar alternativas para aumentar o volume de resíduos alimentares para suprir o biodigestor. Uma opção seria promover a coleta de resíduos da vizinhança, a qual se trata de uma área altamente povoada. O entorno do HGE é composto por vários restaurantes, outros estabelecimentos comerciais e também a Universidade Estadual de Ciências da Saúde de Alagoas (UNCISAL), o que permite inferir que não é uma tarefa impossível, conseguir resíduos alimentares suficientes para tornar a adoção do biodigestor no HGE viável sob o ponto de vista econômico.

De acordo com Lindemeyer (2008), a implantação de uma unidade cogeradora de energia se viabiliza economicamente pelo equivalente em quilowatts/hora evitados no consumo tradicional. A economia é grande quando, por exemplo, a energia gerada é utilizada para suprir a demanda durante o horário de pico (entre as 18 e as 21 horas), em que o custo da eletricidade chega a sete vezes o valor do horário normal.

Utilizar a eletricidade gerada pela biomassa apenas em determinados horários só é possível porque essa fonte, sob esse aspecto, assemelha-se muito a outra renovável, a hidroeletricidade. Assim como a energia é armazenada na forma de água nos reservatórios das usinas hidrelétricas, ela pode ser armazenada na forma de biogás em gasômetros.

Balcezak et al. (2014) conseguiram obter uma economia de a 2,1 milhões de dólares com a inserção de um programa de eficiência energética e manejo racional de resíduos no complexo de serviços de saúde, intitulado "Yale-New Haven Hospital (YNHH)", em Yale, E.U.A. Nesse programa, a principal ação efetiva foi o uso de um biodigestor para todos os resíduos orgânicos do complexo. 
Em termos de condições técnicas e operacionais, a ANEEL (2015), pelo Decreto Federal no ${ }^{\circ}$.163/04 e pela Norma Técnica 167/05, reconheceu e regulamentou a geração de energia próxima ao local de consumo. Com isso abre-se a possibilidade para as unidades de saúde no aproveitamento dos resíduos alimentares.

Góis (2011) destaca que na maioria das instalações com o uso de cogeração energética, principalmente pelo uso biodigestores, a redução da fatura energética nesse sistema é de cerca de $20 \%$ a 30\%, conduzindo a retornos de investimento na ordem dos 3 anos. Os sistemas tradicionais de produção de energia elétrica (centrais termoelétricas e nucleares) têm uma eficiência média da ordem dos $37 \%$, ou seja, apenas $37 \%$ da energia total consumida é convertida em energia elétrica (Silva, 2012). Rios (2008) relata que a eficiência dos sistemas tradicionais de energia elétrica é ainda menor, comparada a sistemas de cogeração de energia a partir de fontes renováveis, quando contabilizadas as perdas por transporte, que podem atingir os $5 \%$, sendo todo o restante perdido para a atmosfera sob a forma de calor.

Vale destacar que a energia elétrica é um dos fatores que mais contribui para a emissão de gases do efeito de estufa nos dias atuais, ou seja, mais de $50 \%$ das emissões de dióxido de carbono $\left(\mathrm{CO}_{2}\right)$ são atribuídas ao consumo de eletricidade nos setores residenciais e edifícios de serviços (Schneider Electric, 2009). Desta maneira, também se pode incluir os hospitais, que são grandes consumidores de eletricidade e também grandes emissores de GEE.

\section{Conclusão}

A adoção de um biodigestor no Hospital Geral de Alagoas nas condições do estudo (número de leitos) permitiria o aproveitamento de 20.914,40 $\mathrm{kg} / \mathrm{ano}$ de resíduos alimentares, gerando anualmente 5.352 KWh de energia. No entanto, o custo do KWh gerado pelo biodigestor é seis vezes maior do que o preço pago atualmente pela Eletrobrás, evidenciando assim que mesmo diante dos benefícios ambientais (aproveitamento dos resíduos e emissão evitada de $\mathrm{CO}_{2}$ ), do ponto de vista econômico a adoção deste sistema ainda não é viável. Porém, se toda a capacidade do biodigestor $\left(10 \mathrm{~m}^{3}\right)$ fosse aproveitada, seria possível gerar $23.000 \mathrm{KWh} / \mathrm{ano}$, a um custo de $\mathrm{R} \$ / \mathrm{KWh} 0,34$, o que é somente $41,0 \%$ superior ao valor pago a Eletrobrás ( $\$$ /KWh 0,24$)$. Estes resultados indicam que caso o HGE se disponha a implantar um programa de recolhimento dos resíduos alimentares da área em seu entorno, a adoção do sistema de biodigestor pode se tornar viável técnica e economicamente, gerando uma energia renovável e limpa.

\section{Referências Bibliográficas}

Balcezak, A. \& Col, B. 2014. Fourth largest hospital in the USA. Disponível em $<$ http://www.ycci.yale.edu/about/ycci/ynhh.as px>. Acesso em 21 de setembro de 2016.

Batista, L. F. 1981. Construçao e operaçao de biodigestores: modelo indiano. Séries manuais. EMBRATER.

Beduschi, L. C., Oortolonai, A. F. \& Coan, O. 2009. Considerações gerais sobre a estação da UNESP de biogás automotivo a baixa pressão. In: EMBRATER (ed.) II encontro sobre biogás automotivo. Jaboticabal.

Bueno, J. 2013. A matriz energética brasileira: situação atual e perspectivas.

Catapan, A., Catapan, D. C. \& Catapan, E. A. 2010. Formas alternativas de geração de energia elétrica a partir do biogás: uma abordagem do custo de geração da energia. Anais do Congresso Brasileiro de Custos$A B C$.

Cervi, R. G., Esperancini, M. S. T. \& Bueno, O. C. 2010. Viabilidade econômica da utilização do biogás produzido em granja suinícola para geração de energia elétrica. Engenharia Agrícola, 30, 831-844.

Deganutti, R., Palhaci, M. C. J. P. \& Rossi, M. 2002. Biodigestores rurais: modelo indiano, chinês e batelada. Procedings of the 4th Encontro de Energia no Meio Rural. Campinas.

Góis, A. F. D. 2011. Demonstração da viabilidade de implementação de uma central de cogeração a um centro informático. Instituto Superior de Engenharia de Lisboa. Lisboa, Lisboa, Portugal.

Lindemeyer, R. M. 2008. Análise da viabilidade econômico-financeira do uso do biogás como fonte de energia elétrica. Santa Catarina.

Lucas Júnior, J. E. P. 2016. Avaliação técnica e econômica de um biodigestor de fluxo tubular: estudo de caso do modelo implantado na etec" orlando quagliato" em Santa Cruz do Rio Pardo, SP, Santa Cruz do Rio Pardo, São Paulo. 
Medeiros, G. A., Caetano, M. M. M., Moraes, F. G., Machado, F. H., Paes, M. X., Amancio, D. T., Ribeiro, A. I., Pinheiro, L. T. \& Ribeiro, L. F. C. 2015. Gestão de resíduos sólidos e biodigestor: abordagem extensionista. Pesquisa em Educação Ambiental, 1, 1-6.

Metz, H. L. 2013. Construção de um biodigestor caseiro para demonstração de produção de biogás e biofertilizante em escolas situadas em meios urbanos. Departamento de Engenharia Rural. Universidade Federal de Lavras, Lavras.

Nogueira, L. A. H. 1992. Biodigestão: A alternativa energética. Nobel, São Paulo.

Pfitscher, E. D., Pfttscher, P. C., Petry, M. P. \& Limogi, B. 2006. Avaliação do Gerenciamento dos Aspectos e Impactos Ambientais de um Hospital. XXIV simpósio de Gestão da Inovação tecnológica. ANPAD, Gramado. Gramado, RS.

RGHVS. 2015. Rede Global de Hospitais Verdes e Saudáveis. Agenda Global para Hospitais Verdes e Saudáveis.

Rios, A. S. 2008. Medidas de Eficiência Energética e Ambiental na Indústria. Vila Real.

Rodrigues, A. C., Baum, C. A., Formentini, J., Bozzetto, C., Ritter, L. G. \& Da Ros, C. O.
2017. Atributos químicos de resíduos orgânicos compostados. Revista Gestão \& Sustentabilidade Ambiental, 6, 193-208.

Santos, E. L., Barbosa, J. H., Melo, M. J., Pereira Junior, D. A., Medeiros, C. S. \& Santos, I. V. V. S. 2017. Uma alternativa energética e ambientalmente sustentável ao agricultor familiar: dia de campo sobre biodigestores rurais. Diversitas Journal, 2, 32-38.

Santos, P. 2000. Guia técnico de biogás. Centro para a Conservação de Energia. Portugal.

Sganzerla, E. 1983. Biodigestor: uma solução. Agropecuária.

Silva, N. F. 2012. Eficiência energética, aplicação em edifícios de serviços: o caso do hospital do ICUF. Instituto Superior de Engenharia do Porto. Instituto Politécnico do Porto, Lisboa.

Sotti, G. 2014. Biogás de digestão anaeróbia dos resíduos orgânicos de restaurante universitário com efluente sanitário. Universidade Tecnológica Federal do Paraná, Londrina, Paraná.

Article History:

Received 8 August 2017

Available on line 24 October 2017

License information: This is an open-access article distributed under the terms of the Creative Commons Attribution License 4.0, which permits unrestricted use, distribution, and reproduction in any medium, provided the original work is properly cited. 